\title{
MS092.005
}

\section{Biodegradable containers based on nanostructured polycrystals obtained by controlled crystallization}

Daria Trushina ${ }^{1}$, Tatiana Bukreeva ${ }^{2}$, Tatiana Borodina ${ }^{3}$, Anna Khovankina ${ }^{4}$, Roman Akasov ${ }^{4}$, Elena Markvicheva ${ }^{4}$ ${ }^{1}$ National Research Centre "Kurchatov Institute", Moscow, Russian Federation, ${ }^{2}$ National Research Centre, Moscow, Russian Federation, ${ }^{3}$ Federal Scientific Research Centre "Crystallography and Photonics" of Russian Academy of Sciences, Moscow, Russian Federation, ${ }^{4}$ M.M. Shemyakin-Yu.A. Ovchinnikov Institute of Bioorganic Chemistry of Russian Academy of Sciences, Moscow, Russian Federation

E-mail: trushina.d@mail.ru

Trushina D.B.1,2, Bukreeva T.V.1,2, Borodina T.N.2, Khovankina A.V.3, Akasov R.A.3, Markvicheva E.A.3

1National Research Centre "Kurchatov Institute", Moscow, Russia, 123182, pl. Kurchatova, 1

2Federal Scientific Research Centre "Crystallography and Photonics" of Russian Academy of Sciences, Moscow, Russia, 119333, Leninskii pr. 59

3M.M. Shemyakin-Yu.A. Ovchinnikov Institute of Bioorganic Chemistry of Russian Academy of Sciences, Moscow, Russia, 117997, GSP-7, Ulitsa Miklukho-Maklaya, 16/10

trushina.d@mail.ru

Multilayer containers fabricated via layer-by-layer sequential deposition of polymers onto a sacrificial core template represent a versatile delivery system for a variety of species used in biomedicine. From a several colloid templates calcium carbonate is of particular interest due to the mild conditions of decomposition. Among the polymorph modifications of calcium carbonate, vaterite particles are favourable due to their porous structure, ability to absorb and release a variety of bioactive compounds. The common $\mathrm{CaCO} 3$ synthesis method of mixing aqueous solutions of calcium chloride and sodium carbonate allows spherical vaterite particles with the size ranging from $5 \mu \mathrm{m}$ to $15 \mu \mathrm{m}$, while drug delivery applications require carriers small enough to penetrate the blood capillaries and cells. So the size of calcium carbonate particles has to be reduced and the main goal of the research is to develop a synthesis method that allows submicron porous vaterite particles in a sizecontrolled manner. Besides, vaterite structure is still under debate what is the best for our knowledge.

To achieve the controlled crystallization of vaterite with desired size a systematic study has been undertaken. The strong influence of mixing time, concentration of reagents and its ratio on the size and structure of calcium carbonate particles was observed. Stable vaterite particles with average diameter 350-500 nm were synthesized after the optimization of the crystallization conditions. It was shown that these spherical particles are formed by attachment of vaterite nanocrystallites. The analysis of the broadening of vaterite diffraction lines reveals the well-defined anisotropic shape of crystallites, which can be described by biaxial ellipsoid with main axes 50 and $100 \mathrm{~nm}$. Owing to such structure submicron vaterite spheres possess a high degree of porosity and therefore good absorption ability that makes them suitable for encapsulation purposes. Surface area of the particles with average size of $500 \mathrm{~nm}$ determined by the method of gas adsorption/desorption amounted to $16,1 \mathrm{~m} 2 / \mathrm{g}$, and the pore size $-30-80 \mathrm{~nm}$.

The obtained particles were used as a template for polymer multilayer capsules comprising 2 and 4-bilayers of biodegradable polyelectrolytes poly-L-arginine and dextran sulfate. In order to confine the capsule size to the nanoscale to meet in vivo requirements, a new approach involving temperature exposure was employed. The heat induced shrinking results in approximately $50 \%$ decrease of average capsule diameter containing both odd and even number of polymer layers. The designed biocompatible carriers with size less than $300 \mathrm{~nm}$ were loaded with doxorubucin. Capsules uptake, toxicity and antitumor activity of encapsulated doxorubucin were studied by means of MTT-test in vitro.

Keywords: Drug delivery, vaterite, polyelectrolyte capsules 